\title{
Error Resilient Pre/Post-Filtering for DCT-Based Block Coding Systems
}

\author{
Chengjie Tu, Member, IEEE, Trac D. Tran, Member, IEEE, and Jie Liang, Member, IEEE
}

\begin{abstract}
Block coding based on the discrete cosine transform (DCT) is very popular in image and video compression. Pre/post-filtering can be attached to a DCT-based block coding system to improve coding efficiency as well as to mitigate blocking artifacts. Previously designed pre/post-filters are optimized to maximize coding efficiency solely. For image and video communication over unreliable channels, those pre/post-filters are sensitive to transmission errors. This paper addresses the problem of designing pre/post-filters which are more error resilient. Reconstruction performance is measured by how low the average reconstruction error is, and how uniformly the reconstruction error is distributed. A family of pre/post-filters is designed to provide desired tradeoffs between coding efficiency and robustness to transmission errors. Experiments show that these filtering operators can achieve superior reconstruction performance without sacrificing much coding performance.
\end{abstract}

Index Terms-Block coding, discrete cosine transform (DCT), error resilience, pre/post-filtering.

\section{INTRODUCTION}

B LOCK coding based on the discrete cosine transform (DCT) [1] is the basis for many international compression standards from JPEG for image coding to the MPEG family and the H26x family for video coding. It enjoys the DCT's excellent energy compaction capability within a data block, low complexity, and high flexibility on a block-by-block basis. Unfortunately, its coding efficiency heavily suffers from ignoring correlation between blocks. More annoyingly, blocking artifacts (reconstruction discontinuities at block boundaries resulting from quantization mismatches) manifest at low bitrates. The problem roots in the fact that DCT-based block coding partitions the signal into nonoverlapped blocks, and each block is transformed and quantized independently.

A remedy to DCT-based block coding is replacing the DCT with a lapped transform (LT) [2]. The key that LTs can greatly improve coding efficiency and reduce blocking artifacts is the overlapping property: Basis functions of LTs are longer than the block size. Analysis filtering extends to the neighboring blocks and correlation between blocks is largely compensated. A data

Manuscript received October 22, 2002; revised September 11, 2004. This research was supported by the National Science Foundation under CAREER Grants CCR-0093262 and CCF-0430869. The associate editor coordinating the review of this manuscript and approving it for publication was Dr. Susie Wee.

C. Tu is with Microsoft Digital Media Division, Microsoft Corporation, Redmond, WA 98052 USA (e-mail: chentu@microsoft.com).

T. D. Tran is with the Department of Electrical and Computer Engineering, The Johns Hopkins University, Baltimore, MD 21218 USA (e-mail: trac@jhu.edu).

J. Liang is with School of Engineering Science, Simon Fraser University, Burnaby, BC V5A 1S6, Canada (e-mail: jiel@sfu.ca).

Digital Object Identifier 10.1109/TIP.2005.860350 block is reconstructed by overlap-adding contributions from several coefficient blocks and quantization mismatches along the boundaries are significantly reduced due to the smoothing effect.

An equivalent but more elegant way to improve DCT-based block coding is recently proposed in [3]: employing pre-filtering in the encoder and post-filtering in the decoder along the block boundaries of the DCT coding framework. Pre/post-filtering combined with the DCT can be viewed as a particular implementation of LTs. Here, the overlapping principle is achieved by pre/post-filtering. Among others, two attractive features of the approach are as follows:

- pre/post-filtering operates in the time domain and is completely outside the existing DCT-based infrastructure;

- the complicated overlapped operation is implemented by two simple stages of nonoverlapped block operations (a pre/post-filtering stage and a DCT/IDCT stage).

It has been demonstrated that with pre/post-filtering, DCT block coding can achieve much better performance at a cost of slightly increased complexity [4], [5].

Due to the growing interest in image/video delivery over unreliable channels such as wireless networks, error-resilient image/video coding has lately received a lot of heavy attention. A comprehensive review on techniques combating transmission errors can be found in [6]. For block coding, it has been established that using LTs instead of the DCT can greatly reduce the visual effect of lost blocks. The reason is that the information of a data block is distributed into several coefficient blocks because of overlapping. Thus, if a coefficient block is lost, partial information of that data block is still available, and the spatial region affected by the lost block increases making the reconstruction error less intensive locally.

For data compression, transforms are generally designed to maximize their coding efficiency under certain complexity and/or regularity constraints. Those transforms are sensitive to transmission errors. In [7], Hemami designed a set of lapped orthogonal transforms (LOTs), $\{\mathrm{T} 6, \mathrm{~T} 7, \mathrm{~T} 8, \mathrm{~T} 9\}$, more immune to transmission errors. Compared to conventional LOTs, although T6-T9 bases do not decrease reconstruction errors on average, errors are distributed across all transform coefficients more uniformly, resulting in visually better reconstructions. The robustness of T6-T9 has been further proven by a multiple description coder presented in [8]. Using the same design method but with a different reconstruction method, Chung and Wang designed another set of error-resilient LOTs [9].

The problem of designing pre/post-filters optimized for coding efficiency has been extensively investigated in [3] and [10]-[12]. This paper concentrates on the design of 


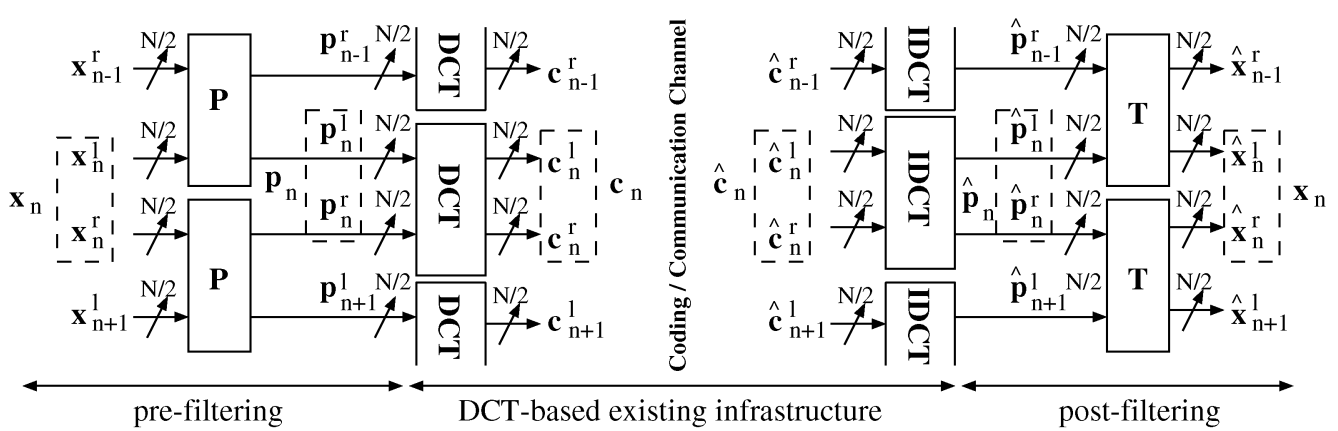

Fig. 1. Pre/post-filtering framework.

error-resilient pre/post-filters. Due to the connection between pre/post-filters and LTs, the paper can be viewed as an extension to Hemami's pioneering work of designing error-resilient LOTs. However, we shall proceed to show that the proposed pre/post-filtering approach provides many advantages over the conventional direct LOT design.

- Fewer free parameters and constraints are involved.

- Pre/post-filter based LTs are more computationally efficient than T6-T9.

- Orthogonality is not imposed since biorthogonal LTs are more desirable because they have higher coding efficiency and are better at eliminating coding artifacts.

- We sometimes do not even impose perfect reconstruction (PR): Several post-filters can be switched between dynamically to improve reconstruction quality.

- Unlike T6-T9, which can only improve error distribution, pre/post-filters can be designed to improve not only error distribution but also notably decrease average error, resulting in improved reconstructions.

The rest of the paper is organized as follows. The next section reviews the pre/post-filtering framework and the relationship between pre/post-filtering and LTs. In Section III, reconstruction criteria are defined and investigated. Error-resilient pre/post-filters are designed in Section IV based on the simple mean reconstruction method. Section V evaluates the performance of the pre/post-filters and the paper is concluded in Section VI.

Notation wise, we use $\mathbf{I}_{N}, \mathbf{J}_{N}, \mathbf{0}_{N}$ to denote the $N \times N$ identity matrix, the $N \times N$ reversal matrix, and the $N \times N$ null matrix, respectively. Also, $[f(i, j)]_{N}$ represents an $N \times N$ matrix with $f(i, j)$ as its element at the $i$ th row and the $j$ th column, where $f(.,$.$) is a certain function. The A R(1)$ image model [13] with unit variance $\sigma_{x}^{2}$ and intersample correlation $\rho=0.95$ is assumed throughout.

\section{Pre/Post-Filtering}

The pre/post-filtering framework [3] is illustrated in Fig. 1: The $N \times N$ pre-filtering block operator $\mathbf{P}$ and the $N \times N$ post-filtering block operator $\mathbf{T}$ operate on the boundaries of the $N$-point DCT without affecting the existing DCT-based infrastructure. We can partition $\mathbf{P}$ and $\mathbf{T}$ into square $(N / 2) \times(N / 2)$ submatrices

$$
\mathbf{P}=\left[\begin{array}{ll}
\mathbf{P}_{00} & \mathbf{P}_{01} \\
\mathbf{P}_{10} & \mathbf{P}_{11}
\end{array}\right], \quad \mathbf{T}=\left[\begin{array}{ll}
\mathbf{T}_{00} & \mathbf{T}_{01} \\
\mathbf{T}_{10} & \mathbf{T}_{11}
\end{array}\right]
$$

In the encoder, the time domain input signal $\mathbf{x}$ is first mapped into the pre-filtered signal $\mathbf{p}$ by $\mathbf{P}$

$$
\left[\begin{array}{l}
\mathbf{p}_{n-1}^{r} \\
\mathbf{p}_{n}^{l}
\end{array}\right]=\mathbf{P}\left[\begin{array}{l}
\mathbf{x}_{n-1}^{r} \\
\mathbf{x}_{n}^{l}
\end{array}\right]=\left[\begin{array}{l}
\mathbf{P}_{00} \mathbf{x}_{n-1}^{r}+\mathbf{P}_{01} \mathbf{x}_{n}^{l} \\
\mathbf{P}_{10} \mathbf{x}_{n-1}^{r}+\mathbf{P}_{11} \mathbf{x}_{n}^{l}
\end{array}\right]
$$

and then the DCT maps $\mathbf{p}$ into the frequency domain signal $\mathbf{c}$ which is then quantized, entropy coded, and transmitted to the decoder

$$
\mathbf{C}_{N} \mathbf{p}_{n}=\mathbf{c}_{n}
$$

where $\mathbf{C}_{N}$ is the $N \times N$ type-II DCT matrix. In the decoder, the IDCT and the post-filter $\mathbf{T}$ perform the inverse operations

$$
\begin{aligned}
\hat{\mathbf{p}}_{n} & =\mathbf{C}_{N}^{-1} \hat{\mathbf{c}}_{n} \\
{\left[\begin{array}{l}
\hat{\mathbf{x}}_{n-1}^{r} \\
\hat{\mathbf{x}}_{n}^{l}
\end{array}\right] } & =\mathbf{T}\left[\begin{array}{l}
\hat{\mathbf{p}}_{n-1}^{r} \\
\hat{\mathbf{p}}_{n}^{l}
\end{array}\right]=\left[\begin{array}{l}
\mathbf{T}_{00} \hat{\mathbf{p}}_{n-1}^{r}+\mathbf{T}_{01} \hat{\mathbf{p}}_{n}^{l} \\
\mathbf{T}_{10} \hat{\mathbf{p}}_{n-1}^{r}+\mathbf{T}_{11} \hat{\mathbf{p}}_{n}^{l}
\end{array}\right]
\end{aligned}
$$

where $\hat{\mathbf{c}}, \hat{\mathbf{p}}, \hat{\mathbf{x}}$ are the received $\mathbf{c}$, the reconstructed $\mathbf{p}$ and $\mathbf{x}$, respectively. Here, $\mathbf{x}$ is segmented into nonoverlapping blocks of length $N$ and the $N$-point column vector $\mathbf{x}_{\mathbf{n}}$ is the $n$th block consisting two $N / 2$-point halves $\mathbf{x}_{n}^{l}$ and $\mathbf{x}_{n}^{r}$, i.e., $\mathbf{x}_{n}=\left[\begin{array}{c}\mathbf{x}_{n}^{l} \\ \mathbf{x}_{n}^{r}\end{array}\right]$. The same convention applies to $\mathbf{p}, \mathbf{c}, \hat{\mathbf{c}}, \hat{\mathbf{p}}$, and $\hat{\mathbf{x}}$. It can be shown [3] that the pre/post-filtering framework generates an $N$-band $2 N$-tap $(N \times 2 N)$ LT with the following forward transform:

$$
\mathbf{H}=\mathbf{C}_{N}\left[\begin{array}{cccc}
\mathbf{P}_{10} & \mathbf{P}_{11} & \mathbf{0}_{\frac{N}{2}} & \mathbf{0}_{\frac{N}{2}} \\
\mathbf{0}_{\frac{N}{2}} & \mathbf{0}_{\frac{N}{2}} & \mathbf{P}_{00} & \mathbf{P}_{01}
\end{array}\right]
$$

and inverse transform

$$
\mathbf{F}=\left[\begin{array}{ll}
\mathbf{T}_{01} & \mathbf{0}_{\frac{N}{2}} \\
\mathbf{T}_{11} & \mathbf{0}_{\frac{N}{2}} \\
\mathbf{0}_{\frac{N}{2}} & \mathbf{T}_{00} \\
\mathbf{0}_{\frac{N}{2}} & \mathbf{T}_{01}
\end{array}\right] \mathbf{C}_{N}^{-1} .
$$

Overlapping is realized by employing pre/post-filtering across DCT blocks.

Although any $N \times N$ matrix can be used as pre/post-filters, we are only interested in pre/post-filters with a specific structure as shown in Fig. 2: two stages of butterflies with an $(N / 2) \times(N / 2)$ matrix between them

$$
\begin{aligned}
& \mathbf{P}=\frac{1}{2}\left[\begin{array}{rr}
\mathbf{I}_{\frac{N}{2}} & \mathbf{J}_{\frac{N}{2}} \\
\mathbf{J}_{\frac{N}{2}} & -\mathbf{I}_{\frac{N}{2}}
\end{array}\right]\left[\begin{array}{rr}
\mathbf{I}_{\frac{N}{2}} & \mathbf{0}_{\frac{N}{2}} \\
\mathbf{0}_{\frac{N}{2}} & \mathbf{V}
\end{array}\right]\left[\begin{array}{rr}
\mathbf{I}_{\frac{N}{2}} & \mathbf{J}_{\frac{N}{2}} \\
\mathbf{J}_{\frac{N}{2}} & -\mathbf{I}_{\frac{N}{2}}
\end{array}\right] \\
& \mathbf{T}=\frac{1}{2}\left[\begin{array}{rr}
\mathbf{I}_{\frac{N}{2}} & \mathbf{J}_{\frac{N}{2}} \\
\mathbf{J}_{\frac{N}{2}} & -\mathbf{I}_{\frac{N}{2}}
\end{array}\right]\left[\begin{array}{rr}
\mathbf{I}_{\frac{N}{2}} & \mathbf{0}_{\frac{N}{2}} \\
\mathbf{0}_{\frac{N}{2}} & \mathbf{U}
\end{array}\right]\left[\begin{array}{rr}
\mathbf{I}_{\frac{N}{2}} & \mathbf{J}_{\frac{N}{2}} \\
\mathbf{J}_{\frac{N}{2}} & -\mathbf{I}_{\frac{N}{2}}
\end{array}\right] .
\end{aligned}
$$



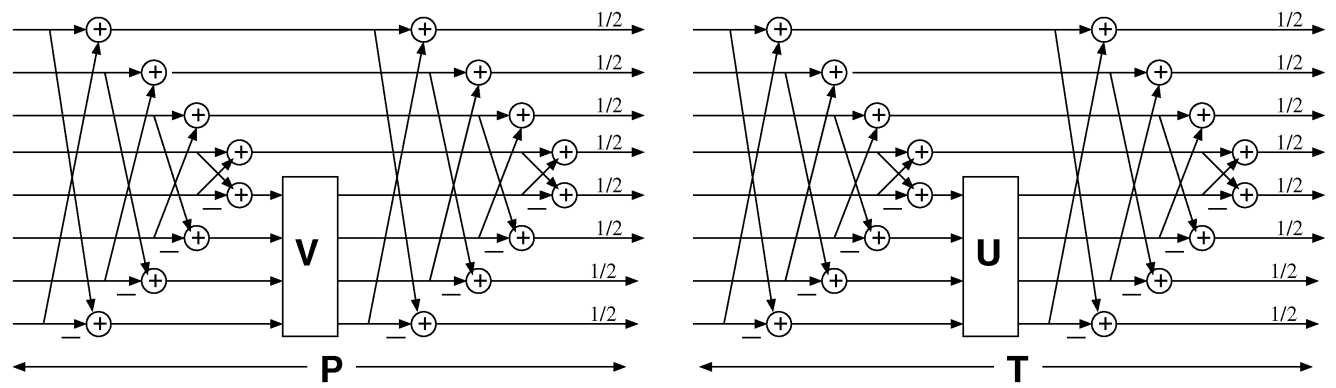

Fig. 2. (Left) Structure of pre-filter and (right) post-filter (drawn for $N=8$ ).

This simple structure offers near optimal coding performance [3]. So, pre/post-filters are uniquely specified by the $(N / 2) \times$ $(N / 2)$ matrices $\mathbf{V}$ and $\mathbf{U}$. Consequently, designing pre/post-filters $\left(N^{2} / 4\right.$ free parameters) is far more tractable than designing LTs directly ( $2 N^{2}$ free parameters). Furthermore, pre/post-filtering is reasonably computationally efficient $\left(N^{2} / 4\right.$ multiplications per block).

With the above structurally imposed solution, we have

$$
\mathbf{P}=\mathbf{J}_{N} \mathbf{P} \mathbf{J}_{N}, \quad \mathbf{T}=\mathbf{J}_{N} \mathbf{T} \mathbf{J}_{N} .
$$

Consequently, rows of $\mathbf{H}$ and $\mathbf{F}$ are either symmetric or antisymmetric, and so linear phase is robustly enforced by the structure [3]. PR requires

$$
\mathbf{U}=\mathbf{V}^{-1} \text {. }
$$

To impose orthogonality, we need

$$
\mathbf{U}=\mathbf{V}^{-1}=\mathbf{V}^{T} \text {. }
$$

By manipulating $\mathbf{V}$ and $\mathbf{U}$, any amount of overlap with a neighboring block up to $N / 2$ samples is possible. For example, if we set $\mathbf{V}=\mathbf{U}=\mathbf{I}_{N / 2}$, then pre/post-filtering is turned off $\left(\mathbf{P}=\mathbf{T}=\mathbf{I}_{N}\right)$ and we go back to the standard DCT-based system. Overlap longer than $N / 2$ samples could be desirable for image coding and error concealment [14]. This can be achieved by using more than one stage of pre/post-filtering, each stage can increase the amount of overlap by $N / 2$ samples. However, this is beyond the scope of this paper.

The coding performance of a pre/post-filter pair is measured via the coding gain of the corresponding LT

$$
G_{T C}=10 \log _{10}\left(\frac{\frac{1}{N} \sum_{i=0}^{N-1} \sigma_{i}^{2}}{\left(\prod_{i=0}^{N-1} \sigma_{i}^{2} f_{i}^{2}\right)^{\frac{1}{N}}}\right)
$$

where $\sigma_{i}^{2}$ and $f_{i}^{2}$ are the $i$ th diagonal entry of $\mathbf{H} \mathcal{R}_{x x} \mathbf{H}^{T}$ and $\mathbf{F}^{T F}$, respectively, whereas $\mathcal{R}_{x x}=\left[\rho^{|i-j|}\right]_{2 N}$ is the autocorrelation matrix of the input signal. To compute the coding gain, we always assume $\operatorname{PR}\left(\mathbf{U}=\mathbf{V}^{-1}\right)$. If the orthogonality condition holds, then $f_{i}^{2}=1$ and (13) reduces to

$$
G_{T C}=10 \log _{10}\left(\frac{\frac{1}{N} \sum_{i=0}^{N-1} \sigma_{i}^{2}}{\left(\prod_{i=0}^{N-1} \sigma_{i}^{2}\right)^{\frac{1}{N}}}\right) .
$$

A significant observation is that the coding gain is not that sensitive to the pre/post-filter choices as long as $\mathbf{V}$ is well-posed (both $\mathbf{V}$ and $\mathbf{V}^{-1}$ are far from singular). One reason for this is that energy compaction is mainly done by the DCT, which has near-optimal energy compaction within the data block; pre/post-filtering only affects the coding gain moderately by taking away correlation between blocks. So, we can often vary $\mathbf{V}$ to achieve other desired properties without sacrificing much coding efficiency.

\section{RECONSTRUCTION CRITERIA}

The imperfect transmission of an $N$-point coefficient block $\mathbf{c}_{n}$ affects the reconstruction of a $2 N$-point signal block $\left[\left(\mathbf{x}_{n-1}^{r}\right)^{T},\left(\mathbf{x}_{n}\right)^{T},\left(\mathbf{x}_{n+1}^{l}\right)^{T}\right]^{T}$. Let us define the reconstruction error $\mathbf{e}_{n}$ and its auto correlation matrix $\mathcal{R}_{e e}$ as

$$
\begin{aligned}
\mathbf{e}_{n} & =\left[\begin{array}{l}
\hat{\mathbf{x}}_{n-1}^{r} \\
\hat{\mathbf{x}}_{n} \\
\hat{\mathbf{x}}_{n+1}^{l}
\end{array}\right]-\left[\begin{array}{l}
\mathbf{x}_{n-1}^{r} \\
\mathbf{x}_{n} \\
\mathbf{x}_{n+1}^{l}
\end{array}\right] \\
\mathcal{R}_{e e} & =E\left\{\mathbf{e}_{n} \mathbf{e}_{n}^{T}\right\} .
\end{aligned}
$$

The $i \operatorname{th}(i=0, \cdots, 2 N-1)$ diagonal entry of $\mathcal{R}_{e e}, e_{i}^{2}$, is the mean-squared error (MSE) of the $i$ th reconstructed sample of the block.

The expected reconstruction quality is fully specified by $e_{i}^{2}$. The objective quality of the reconstructed block in terms of the peak signal-to-noise ratio (PSNR) depends on the MSE for the entire block

$$
\mathrm{MSE}=\frac{1}{2 N} \sum_{i=0}^{2 N-1} e_{i}^{2}=\frac{1}{2 N} \operatorname{Tr}\left(\mathcal{R}_{e e}\right) .
$$

Obviously, a reconstructed block with a smaller MSE has a higher PSNR.

Besides the MSE, the distribution of $e_{i}^{2}$ also has a significant impact on the visual quality of the reconstructed image. A more uniform error distribution has less artifacts and is visually more pleasing. The reconstruction gain can be defined as

$$
G_{R}=\frac{\left(\prod_{i=0}^{2 N-1} e_{i}^{2}\right)^{\frac{1}{2 N}}}{\frac{1}{2 N} \sum_{i=0}^{2 N-1} e_{i}^{2}}
$$

which measures how uniform the distribution is. A more uniform error distribution results in a larger $G_{R}$. The $G_{R}$ achieves its maximum value of 1 when we have a completely 
uniform error distribution, i.e., $e_{i}^{2}$ is expected to be the same for every reconstructed sample. Note that $G_{R}$ is defined in the time domain since visual quality is directly evaluated in the time domain.

If the block post-filtering operation in (5) does not involve any lost coefficients, PR is always preferred $\left(\mathbf{U}=\mathbf{V}^{-1}\right)$. However, if (5) does involve lost coefficients, a post-filter that is not a perfect inverse of the pre-filter $\left(\mathbf{U} \neq \mathbf{V}^{-1}\right)$ may produce better reconstruction quality. Since the decoder knows the locations of the lost coefficients, online adaptive switching between the post-filters according to the availability of the coefficients can be trivially achieved in the pre/post-filtering framework.

\section{Optimal Pre/Post-FILTER Design}

From the previous section, an $N \times N$ pre/post-filter pair is uniquely specified by two $(N / 2) \times(N / 2)$ matrices: $\mathbf{V}$ associated with the pre-filter and $\mathbf{U}$ associated with the post-filter. Designing an error-resilient pre/post-filter pair is equivalent to finding a $\mathbf{V} / \mathbf{U}$ combination to optimize the corresponding $G_{T C}, \mathrm{MSE}$, and $G_{R}$. Unfortunately, $G_{T C}$, MSE, and $G_{R}$ can not reach their optimal values simultaneously. We typically set up an optimization procedure to maximize a weighted sum, $G_{T C}-\alpha \times 10 \log _{10}(\mathrm{MSE})+\beta \times 10 \log 10\left(G_{R}\right)$, taking $\mathbf{V}$ and $\mathbf{U}$ as arguments, where $\alpha$ and $\beta$ are nonnegative weights. The choice of the weights highly depends on the loss pattern. For example, we should bias $G_{T C}$ more if a small percentage of coefficients was lost.

The key of the design procedure is to find the error autocorrelation matrix $\mathcal{R}_{e e}$ according to the specific loss pattern and the recovery method. Without loss of generality, we assume the same simple scenario in [7]: A coefficient block is either lost or received completely, and a lost block is recovered by the mean of its perfectly received neighboring blocks

$$
\hat{\mathbf{c}}_{n}=\frac{1}{2}\left(\mathbf{c}_{n-1}+\mathbf{c}_{n+1}\right) .
$$

This mean reconstruction method has been proven to be effective [15]. Other reconstruction methods do not affect the design procedure; only $\mathcal{R}_{e e}$ might take a different form.

Under the pre/post-filtering framework, (19) is equivalent to

$$
\hat{\mathbf{p}}(n)=\frac{1}{2}\left(\mathbf{p}_{n-1}+\mathbf{p}_{n+1}\right)
$$

and so the recovery can be performed in the time domain after the IDCT.

If we define

$$
\begin{aligned}
& \mathbf{A}=\left[\begin{array}{l}
\mathbf{T}_{01} \\
\mathbf{T}_{11}
\end{array}\right]\left[\begin{array}{ll}
\mathbf{P}_{10} & \mathbf{P}_{11}
\end{array}\right] \\
& \mathbf{B}=\left[\begin{array}{l}
\mathbf{T}_{00} \\
\mathbf{T}_{10}
\end{array}\right]\left[\begin{array}{ll}
\mathbf{P}_{00} & \mathbf{P}_{01}
\end{array}\right] \\
& \mathbf{C}=\mathbf{I}_{N}-\mathbf{A} \\
& \mathbf{D}=\mathbf{I}_{N}-\mathbf{B}
\end{aligned}
$$

it is straightforward to show that the error autocorrelation matrix $\mathcal{R}_{e e}$ is given by

$$
\begin{aligned}
\mathcal{R}_{e e}= & E\left\{\mathbf{e}_{n} \mathbf{e}_{n}^{T}\right\} \\
= & {\left[\begin{array}{rr}
\mathbf{D} & \mathbf{0}_{N} \\
\mathbf{0}_{N} & \mathbf{C}
\end{array}\right]\left[\begin{array}{ll}
\mathcal{R}_{0} & \mathcal{R}_{1}^{T} \\
\mathcal{R}_{1} & \mathcal{R}_{0}
\end{array}\right]\left[\begin{array}{ll}
\mathbf{D}^{T} & \mathbf{0}_{N} \\
\mathbf{0}_{N} & \mathbf{C}^{T}
\end{array}\right] } \\
& -\left[\begin{array}{rr}
\mathbf{A} & \mathbf{0}_{N} \\
\mathbf{0}_{N} & \mathbf{B}
\end{array}\right]\left[\begin{array}{ll}
\mathcal{R}_{2} & \mathcal{R}_{3}^{T} \\
\mathcal{R}_{3} & \mathcal{R}_{2}
\end{array}\right]\left[\begin{array}{ll}
\mathbf{D}^{T} & \mathbf{0}_{N} \\
\mathbf{0}_{N} & \mathbf{C}^{T}
\end{array}\right] \\
& -\left[\begin{array}{rr}
\mathbf{D} & \mathbf{0}_{N} \\
\mathbf{0}_{N} & \mathbf{C}
\end{array}\right]\left[\begin{array}{ll}
\mathcal{R}_{2} & \mathcal{R}_{3}^{T} \\
\mathcal{R}_{3} & \mathcal{R}_{2}
\end{array}\right]\left[\begin{array}{ll}
\mathbf{A}^{T} & \mathbf{0}_{N} \\
\mathbf{0}_{N} & \mathbf{B}^{T}
\end{array}\right] \\
& +\left[\begin{array}{rr}
\mathbf{A} & \mathbf{0}_{N} \\
\mathbf{0}_{N} & \mathbf{B}
\end{array}\right]\left[\begin{array}{ll}
\mathcal{R}_{4} & \mathcal{R}_{5}^{T} \\
\mathcal{R}_{5} & \mathcal{R}_{4}
\end{array}\right]\left[\begin{array}{ll}
\mathbf{A}^{T} & \mathbf{0}_{N} \\
\mathbf{0}_{N} & \mathbf{B}^{T}
\end{array}\right]
\end{aligned}
$$

where $\mathcal{R}_{0}=\left[\rho^{|i-j|}\right]_{N}, \mathcal{R}_{1}=\left[\rho^{N+i-j}\right]_{N}, \mathcal{R}_{2}=1 / 2\left(\mathcal{R}_{1}+\right.$ $\left.\mathcal{R}_{1}^{T}\right), \mathcal{R}_{3}=1 / 2\left(\mathcal{R}_{0}+\rho^{N} \mathcal{R}_{1}\right), \mathcal{R}_{4}=1 / 2\left(\mathcal{R}_{0}+\rho^{N} \mathcal{R}_{2}\right)$, and $\mathcal{R}_{5}=(1 / 2) \mathcal{R}_{1}+(1 / 4)\left(\rho^{2 N} \mathcal{R}_{1}+\mathcal{R}_{1}^{T}\right)$.

If the PR condition $\mathbf{U}=\mathbf{V}^{-1}$ is assumed, then we have $\mathbf{A}+\mathbf{B}=\mathbf{I}_{N}$ and (22) reduces to

$$
\mathcal{R}_{e e}=\left[\begin{array}{rr}
\mathbf{A} & \mathbf{0}_{N} \\
\mathbf{0}_{N} & \mathbf{B}
\end{array}\right] \mathcal{R}_{\mathrm{err}}\left[\begin{array}{ll}
\mathbf{A}^{T} & \mathbf{0}_{N} \\
\mathbf{0}_{N} & \mathbf{B}^{T}
\end{array}\right]
$$

where the $2 N \times 2 N$ matrix

$$
\begin{aligned}
\mathcal{R}_{\mathrm{err}}=\left[\frac{3}{2} \rho^{|i-j|}-\rho^{|N+i-j|}-\rho^{|N+j-i|}\right. \\
\left.+\frac{\rho^{2 N+i-j}+\rho^{2 N+j-i}}{4}\right]_{2 N}
\end{aligned}
$$

is consistent with the definition in [7]. This is a direct consequence of using the same scenario. Noting that the top-left quadrant and the right-bottom quadrant of $\mathcal{R}_{\text {err }}$ are the same matrix denoted as $\mathcal{E}$, and coupling with the fact that $\operatorname{Tr}(\mathbf{X Y})=$ $\operatorname{Tr}(\mathbf{Y X})$, we have

$$
\begin{aligned}
& \mathrm{MSE}=\frac{1}{2 N} \operatorname{Tr}\left(\mathcal{R}_{e e}\right)=\frac{1}{2 N} \operatorname{Tr}\left(\left(\mathbf{A}^{T} \mathbf{A}+\mathbf{B}^{T} \mathbf{B}\right) \mathcal{E}\right)
\end{aligned}
$$

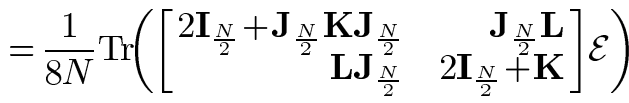

where $\mathbf{K}=\mathbf{V}^{-T} \mathbf{V}^{-1}+\mathbf{V}^{T} \mathbf{V}$, and $\mathbf{L}=\mathbf{V}^{-T} \mathbf{V}^{-1}-\mathbf{V}^{T} \mathbf{V}$. If the orthogonality condition $\mathbf{V}^{-1}=\mathbf{V}^{T}$ is also assumed, then $\mathbf{K}=2 \mathbf{I}_{N / 2}$ and $\mathbf{L}=\mathbf{0}_{N / 2}$, and since $\operatorname{Tr}(\mathcal{E})=(1 / 2) \operatorname{Tr}\left(\mathcal{R}_{\text {err }}\right)$, (25) reduces to

$$
\mathrm{MSE}=\frac{1}{4 N} \operatorname{Tr}\left(\mathcal{R}_{\mathrm{err}}\right) .
$$

So, if orthogonality is imposed, a particular pre/post-filter pair does not affect the MSE of the entire signal block. Noticing that an orthogonal transform does not change the MSE of $e(n)$, this is the exact analogy of Hemami's conclusion that the particular LOT does not affect the MSE of the entire coefficient block [7]. However, this is not true anymore if orthogonality is absent, and some $\mathbf{V} / \mathbf{U}$ pairs may give significant lower MSEs, resulting in much better reconstructions.

It is worth pointing out that our design procedure does not involve the DCT. This greatly simplifies the computation of $\mathcal{R}_{e e}$. Moreover, with the help of (10), it is straightforward to show that $\mathbf{B}=\mathbf{J}_{N} \mathbf{A} \mathbf{J}_{N}$ and $\mathbf{C}=\mathbf{J}_{N} \mathbf{D} \mathbf{J}_{N}$. Next, since $\mathcal{R}_{0}=\mathbf{J}_{N} \mathcal{R}_{0} \mathbf{J}_{N}$, 
$\mathcal{R}_{2}=\mathbf{J}_{N} \mathcal{R}_{2} \mathbf{J}_{N}$, and $\mathcal{R}_{4}=\mathbf{J}_{N} \mathcal{R}_{4} \mathbf{J}_{N}$, if we denote the top-left and bottom-right quadrant of $\mathcal{R}_{e e}$ as $\mathcal{U}$ and $\mathcal{L}$, respectively, then

$$
\begin{aligned}
\mathcal{L}= & \left(\mathbf{C} \mathcal{R}_{0}-\mathbf{B} \mathcal{R}_{2}\right) \mathbf{C}^{T}-\left(\mathbf{C} \mathcal{R}_{2}-\mathbf{B} \mathcal{R}_{4}\right) \mathbf{B}^{T} \\
= & \mathbf{J}_{N}\left(\left(\mathbf{D} \mathbf{J}_{N} \mathcal{R}_{0} \mathbf{J}_{N}-\mathbf{A} \mathbf{J}_{N} \mathcal{R}_{2}\right) \mathbf{J}_{N} \mathbf{D}^{T}\right. \\
& \left.\quad-\left(\mathbf{D} \mathbf{J}_{N} \mathcal{R}_{2} \mathbf{J}_{N}-\mathbf{A} \mathbf{J}_{N} \mathcal{R}_{4}\right) \mathbf{J}_{N} \mathbf{A}^{T}\right) \mathbf{J}_{N} \\
= & \mathbf{J}_{N} \mathcal{U} \mathbf{J}_{N} ;
\end{aligned}
$$

so the diagonal entries of $\mathcal{R}_{e e}$ are even symmetric, i.e., $e_{2 N-1-i}^{2}=e_{i}^{2}(i=0,1, \ldots, N-1)$ and $e_{i}^{2}(i=0,1, \ldots, N-1)$ can be computed as

$$
e_{i}^{2}=\left(\mathbf{D}_{i .} \mathcal{R}_{0}-\mathbf{A}_{i .} \mathcal{R}_{2}\right) \mathbf{D}_{i .}^{T}+\left(\mathbf{A}_{i .} \mathcal{R}_{4}-\mathbf{D}_{i .} \mathcal{R}_{2}\right) \mathbf{A}_{i .}^{T}
$$

where we use $\mathbf{A}_{i}$ and $\mathbf{D}_{i}$. to denote the $i$ th row of $\mathbf{A}$ and $\mathbf{D}$, respectively. If $\mathrm{PR}$ is desired, (28) can be shown to be reduced to $e_{i}^{2}=\mathbf{A}_{i .} \mathcal{E} \mathbf{A}_{i .}^{T}$. It is not necessary to compute $\mathcal{R}_{e e}$ explicitly, and only a small number of multiplications (around $4 N^{3}$, or $N^{3}$ if $\mathrm{PR}$ is assumed) is needed to compute all $e_{i}^{2}$.

Prior to a more practical example $(\mathrm{N}=8)$, we first study a simple illustrative example $(\mathrm{N}=2)$ to shed some lights on how the pre- and post-filters behave.

\section{A. Case $N=2$ With $P R$}

In this case, both $\mathbf{V}=v$ and $\mathbf{U}=1 / v$ are scalars. So, $G_{T C}$, MSE, and $G_{R}$ are one-dimensional functions of $v$. Fig. 3 depicts the behavior of $G_{T C}$, MSE and $G_{R}$ for this example. The first observation is that $G_{T C}, \mathrm{MSE}$ and $G_{R}$ obviously can not achieve their optimal values simultaneously. Second, we always prefer a positive $v$ since $\operatorname{MSE}(|v|)=\operatorname{MSE}(-|v|)$ and $G_{R}(|v|)=G_{R}(-|v|)$ (MSE and $G_{R}$ are even symmetric about $v$ ) while $G_{T C}(|v|)>G_{T C}(-|v|)$. Third, both $|v|$ and $1 /|v|$ should be far from 0 since otherwise $G_{T C}$ will be small and MSE will be large. Finally, there is a large range of $v(v>$ 1 ) where $G_{T C}$ is not very sensitive, and, thus, we can vary $v$ to achieve better MSE and/or $G_{R}$ without adversely affecting $G_{T C}$.

When $N>2$, experiments show that the aforementioned observations still hold when determinant of $\mathbf{V}$ and $|\mathbf{V}|$ takes the place of $|v|$. So, in the optimization procedure, we only consider the pre/post-filters with $|\mathbf{V}|<0$ or $|\mathbf{V}| \approx 0$ or $1 /|\mathbf{V}| \approx 0$. This greatly improves the convergence time as well as the robustness of the optimization process.

\section{B. $N=8$}

In this case, the optimization involves 32 (16 if PR is assumed) free parameters, and the MATLAB function fminsearch can quickly approach a near optimal solution. Table I tabulates several pre/post-filter designs and compares the resulting LTs, $\{\mathrm{P} 1, \mathrm{P} 2, \mathrm{P} 3, \mathrm{P} 4\}$, with several other existing transforms: the DCT, the LOT with the known maximum coding gain $9.22 \mathrm{~dB}$ $\left(\mathrm{LOT}_{\mathrm{opt}}\right)$ [3], the biorthogonal LT with the known maximum coding gain $9.61 \mathrm{~dB}\left(\mathrm{LT}_{\text {opt }}\right)$ [3], and T6 and T9 in [7] (we skip $\mathrm{T} 7$ and $\mathrm{T} 8$ because they give performance between T6 and T9). All transforms except for T6 and T9 fit in the pre/post-filtering framework. P1 achieves the lowest MSE, while P2 and P3 bias coding performance more. $\mathrm{PR}$ is not assumed for $\mathrm{P} 3$ and $\mathrm{P} 4$, and online dynamic switching as described in Section III is neces-
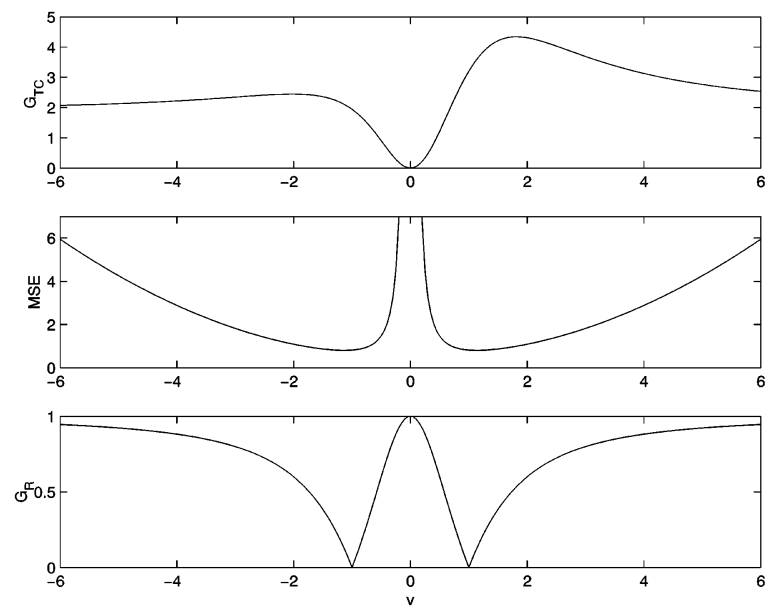

Fig. 3. (Top) Behavior of $G_{T C}$, (middle) MSE, and (bottom) $G_{R}$ in the case of $N=2$ with PR $(\mathbf{V}=v$ and $\mathbf{U}=1 / v)$.

sary. The only difference between $\mathrm{P} 4$ and the $\mathrm{LT}_{\mathrm{opt}}$ is that $\mathrm{P} 4$ can switch post-filters.

As expected, all orthogonal transforms (the DCT, the $\mathrm{LOT}_{\mathrm{opt}}$, T6, and T9) have the same MSE; yet, coding gains and reconstruction gains vary widely. With the DCT, $G_{R}=0$ and the ill error distribution results in severe blocking artifacts. T6 and T9 have better reconstruction gains than the DCT and the $\mathrm{LOT}_{\mathrm{opt}}$, but with much worse coding gains.

Compared to the orthogonal transforms, none of our designs (P1-P4) is orthogonal, but each can achieve a lower MSE and/or a higher $G_{R}$ without heavily sacrificing coding gain. Note that $\mathrm{P} 4$ has the same pre-filter, and, thus, the same coding gain as the $\mathrm{LT}_{\text {opt }}$, while by dynamically switching the post-filter, it has a lower MSE and a much higher $G_{R}$.

Fig. 4 shows the actual distributions of $e_{i}^{2}$ for selected transforms. For transforms with low reconstruction gains (the DCT and the $\mathrm{LT}_{\mathrm{opt}}$ ), the errors concentrate on the center 8-point block, while, for the others, errors are distributed to other pixels. Clearly, $\mathrm{P} 4$ has a more uniform error distribution than the $\mathrm{LT}_{\mathrm{opt}}$ does. Finally, note that on average the error distribution curve for P1 is well under the others since P1 has a far lower MSE.

\section{TRAnSform Performance}

Accurately simulating a real image communication system is beyond the scope of this paper. We benchmark transform performance using the following simple setup: Positions of lost blocks are known to the decoder and the coded information of a block is assumed to be packetized into one packet. We test the following five block loss patterns.

- S0: No loss.

- S1: 25\% regular loss as shown on the left of Fig. 5.

- S2: 50\% regular loss depicted in the middle of Fig. 5.

- S3: $25 \%$ random loss.

- S4: $50 \%$ random loss.

For S3 and S4, we repeatedly discard the information of the block at a random position until enough blocks are lost. Scenario S0 corresponds to perfect channels; S1 and S2 occur in multiple description coding where an image is coded as several independent descriptions; and S3 and S4 simulate the case that an 
TABLE I

PRE/Post-FILTER DESIGNS AND COMPARISONS BETWEEN THE RESUlting LTS AND SEVERAL OthER STANDARD TRANSFORMS

\begin{tabular}{|c|c|c|c|c|c|c|c|c|c|c|c|}
\hline Transform & \multicolumn{4}{|c|}{$\mathbf{V}$} & \multicolumn{4}{|c|}{$\mathbf{U}$} & $G_{T C}$ & $M S E$ & $\overline{G_{R}}$ \\
\hline DCT & \multicolumn{4}{|c|}{$\mathbf{I}_{4}$} & \multicolumn{4}{|c|}{$\mathbf{I}_{4}$} & 8.31 & 0.200 & 0 \\
\hline $\mathrm{LOT}_{o p t}$ & $\begin{array}{r}0.8072 \\
-0.5718 \\
0.1218 \\
-0.0814\end{array}$ & $\begin{array}{r}0.5594 \\
0.6992 \\
-0.4443 \\
-0.0286 \\
\end{array}$ & $\begin{array}{r}0.1436 \\
0.4214 \\
0.8600 \\
-0.2492 \\
\end{array}$ & $\begin{array}{l}0.1218 \\
0.0814 \\
0.2193 \\
0.9646\end{array}$ & \multicolumn{4}{|c|}{$\mathbf{V}^{T}$} & 9.22 & 0.200 & 0.44 \\
\hline $\mathrm{LT}_{o p t}$ & {$\left[\begin{array}{r}0.9550 \\
-0.5520 \\
0.1123 \\
-0.0295\end{array}\right.$} & $\begin{array}{r}0.7833 \\
0.9008 \\
-0.3646 \\
0.0081\end{array}$ & $\begin{array}{r}0.3548 \\
0.6188 \\
1.0916 \\
-0.1196\end{array}$ & $\begin{array}{l}0.2391 \\
0.2354 \\
0.3904 \\
1.1879\end{array}$ & \multicolumn{4}{|c|}{$\mathbf{V}^{-1}$} & 9.61 & 0.212 & 0.37 \\
\hline T6 & \multicolumn{4}{|c|}{ Not Applicable } & \multicolumn{4}{|c|}{ Not Applicable } & 7.83 & 0.200 & 0.55 \\
\hline T9 & \multicolumn{4}{|c|}{ Not Applicable } & \multicolumn{4}{|c|}{ Not Applicable } & 6.50 & 0.200 & 0.85 \\
\hline $\mathrm{P} 1$ & {$\left[\begin{array}{r}-1.6769 \\
-0.7091 \\
-0.1774 \\
-0.1131\end{array}\right.$} & $\begin{array}{l}0.6005 \\
1.2843 \\
0.7553 \\
0.1046\end{array}$ & $\begin{array}{l}-0.3369 \\
-0.4077 \\
-1.1195 \\
-0.8291\end{array}$ & $\begin{array}{l}0.1006 \\
0.1601 \\
0.1202 \\
0.9090\end{array}$ & \multicolumn{4}{|c|}{$\mathbf{V}^{-1}$} & 6.95 & 0.140 & 0.67 \\
\hline $\mathrm{P} 2$ & $\begin{array}{r}0.5183 \\
0.1582 \\
1.1711 \\
-0.0511\end{array}$ & $\begin{array}{r}-0.3612 \\
0.8663 \\
0.2693 \\
0.2264 \\
\end{array}$ & $\begin{array}{l}-1.2530 \\
-1.2547 \\
-0.4468 \\
-0.2225\end{array}$ & $\begin{array}{l}0.8415 \\
0.5062 \\
0.4451 \\
0.9502 \\
\end{array}$ & \multicolumn{4}{|c|}{$\mathbf{V}^{-1}$} & 8.41 & 0.153 & 0.64 \\
\hline P3 & {$\left[\begin{array}{r}0.6554 \\
-0.5047 \\
0.5262 \\
-0.0742\end{array}\right.$} & $\begin{array}{r}0.8603 \\
0.5498 \\
-0.4003 \\
0.1439\end{array}$ & $\begin{array}{r}-0.0125 \\
0.6950 \\
0.8138 \\
-0.0503\end{array}$ & $\begin{array}{r}-0.1330 \\
-0.2457 \\
0.1117 \\
0.9080\end{array}$ & {$\left[\begin{array}{r}\text { sw } \\
0.3200 \\
0.2222 \\
-0.1779 \\
-0.0801\end{array}\right.$} & $\begin{array}{c}\text { hing bet } \\
-0.5082 \\
0.0874 \\
0.1938 \\
0.0443\end{array}$ & $\begin{array}{r}\text { een } \mathbf{V}^{-1} \\
0.5482 \\
-0.2836 \\
0.1714 \\
-0.3094\end{array}$ & $\begin{array}{c}\text { and } \\
-0.2454 \\
0.3959 \\
0.4531 \\
1.1220\end{array}$ & 9.17 & 0.161 & 0.59 \\
\hline $\mathrm{P} 4$ & {$\left[\begin{array}{r}0.9550 \\
-0.5520 \\
0.1123 \\
-0.0295\end{array}\right.$} & $\begin{array}{r}0.7833 \\
0.9008 \\
-0.3646 \\
0.0081\end{array}$ & $\begin{array}{r}0.3548 \\
0.6188 \\
1.0916 \\
-0.1196\end{array}$ & $\begin{array}{l}0.2391 \\
0.2354 \\
0.3904 \\
1.1879\end{array}$ & {$\left[\begin{array}{r}\text { sw } \\
0.3860 \\
0.1654 \\
0.0530 \\
-0.0848\end{array}\right.$} & $\begin{array}{r}\text { hing bet } \\
-0.4191 \\
0.2505 \\
-0.0054 \\
0.0348\end{array}$ & $\begin{array}{r}\text { een } \mathbf{V}^{-1} \\
0.2367 \\
-0.1447 \\
0.2076 \\
-0.2124\end{array}$ & $\begin{array}{l}\text { and } \\
-0.1314 \\
-0.0100 \\
0.2568 \\
0.7750\end{array}$ & 9.61 & 0.209 & 0.62 \\
\hline
\end{tabular}

TABLE II

RECONSTRUCTION RESULTS IN TERMS OF PSNR (WITHOUT QUANTIZATION) UNDER VARIOUS LOSS PATTERNS

\begin{tabular}{c||c|c|c|c||c|c|c|c}
\hline \multicolumn{1}{c||}{ Transform } & S1 & S2 & S3 & S4 & S1 & S2 & S3 & S4 \\
\hline \hline \multicolumn{1}{l||}{} & \multicolumn{9}{c||}{ Lena } & \multicolumn{4}{c}{ Barbara } \\
\hline DCT & 27.41 & 24.26 & 26.30 & 22.64 & 26.62 & 23.49 & 26.00 & 21.65 \\
\hline LOT $_{\text {opt }}$ & 27.42 & 24.23 & 26.32 & 22.66 & 26.61 & 23.48 & 26.01 & 21.64 \\
\hline LT $_{\text {opt }}$ & 27.07 & 23.91 & 26.11 & 22.59 & 26.53 & 23.18 & 25.85 & 21.49 \\
\hline \hline T6 & 27.40 & 24.23 & 26.38 & 22.68 & 26.60 & 23.55 & 25.99 & 21.74 \\
\hline T9 & 27.30 & 24.33 & 26.51 & 22.67 & 26.59 & 23.42 & 25.93 & 21.66 \\
\hline \hline P1 & $\mathbf{3 0 . 8 8}$ & $\mathbf{2 6 . 7 3}$ & $\mathbf{2 9 . 2 3}$ & $\mathbf{2 4 . 8 5}$ & $\mathbf{2 8 . 0 9}$ & $\mathbf{2 4 . 8 4}$ & $\mathbf{2 7 . 2 6}$ & $\mathbf{2 2 . 9 7}$ \\
\hline P2 & 29.79 & 26.15 & 28.35 & 24.32 & 26.75 & 23.39 & 21.97 & 22.17 \\
\hline P3 & 28.57 & 25.38 & 27.34 & 23.56 & 27.04 & 23.87 & 26.46 & 22.27 \\
\hline P4 & 27.15 & 24.05 & 26.30 & 22.90 & 26.19 & 23.02 & 25.82 & 21.65 \\
\hline \multicolumn{1}{|c|}{}
\end{tabular}
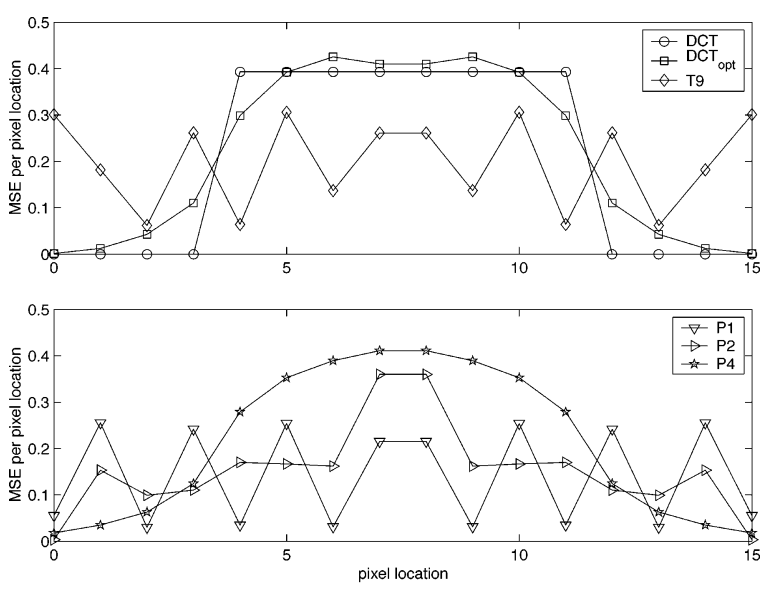

Fig. 4. Error distribution.

image is coded in a single description and transmitted through a channel with random packet loss. Large contiguous areas of

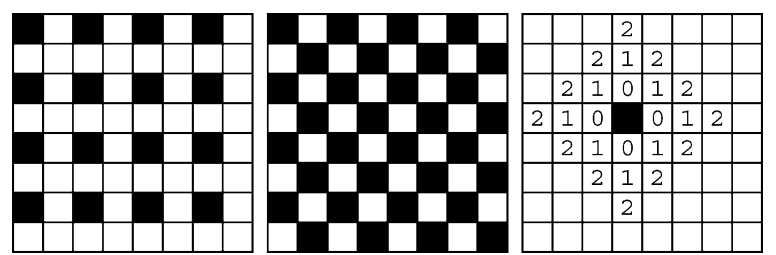

Fig. 5. (Left) 25\% regular block loss, (middle) $50 \%$ regular block loss, and (right) layers of neighbors where " 0 ," "1," and "2" label the first layer, second layer, and third layer, respectively. Black blocks are lost blocks.

loss may happen for S3 and S4. To reconstruct a lost block, we search the nearest layer of neighbors with at least one received block in a diamond order as shown on the right of Fig. 5. In other words, blocks in layer $n+1$ are never considered unless all blocks in layer $n$ are lost. The block is reconstructed as the mean of the received blocks of that layer. The 8-bit $512 \times 512$ Lena and Barbara images are used as the test images. 

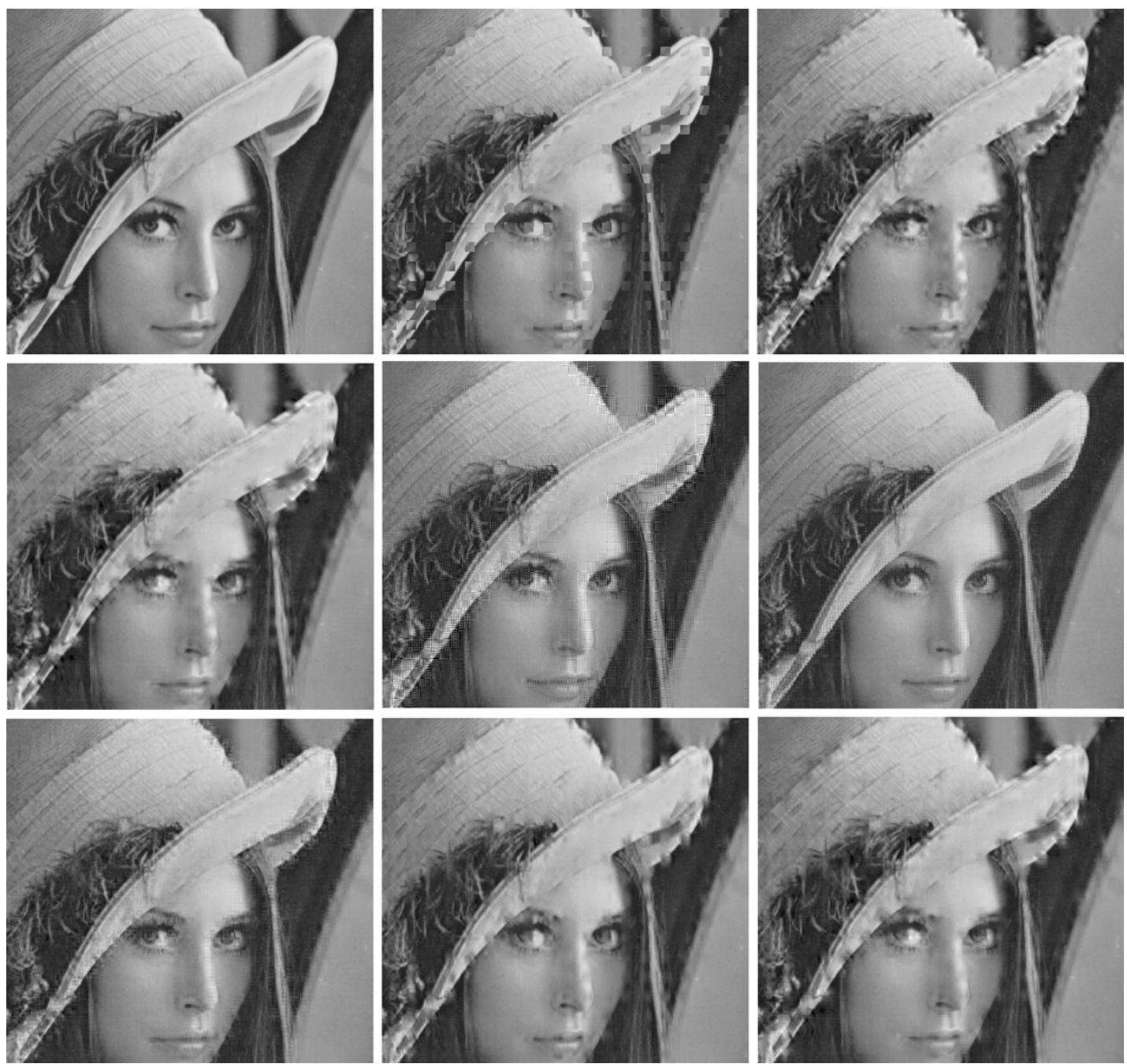

Fig. 6. Portions of reconstructed Lena image without quantization, suffering from $25 \%$ regular loss. From left to right, top row: original; DCT (27.41 dB); LOT $_{\text {opt }}(27.42 \mathrm{~dB})$. Middle row: LOT opt $(27.07 \mathrm{~dB})$; T9 (27.30 dB); P1(30.88 dB). Bottom row: P2 (29.79 dB); P3 (28.57 dB); P4 (27.15 dB).

TABLE III

RECONSTRUCTION RESUlTS IN TERMS OF PSNR (CODED AT 1 bpp BY L-CEB) UNDER VARIOUS LOSS PATTERnS

\begin{tabular}{c||c|c|c|c|c||c|c|c|c|c|c}
\hline Transform & S0 & S1 & S2 & S3 & S4 & S0 & S1 & S2 & S3 & S4 \\
\hline \hline \multicolumn{1}{|c||}{} & \multicolumn{9}{|c||}{ Lena } \\
\hline DCT & 39.78 & 27.24 & 24.22 & 26.18 & 22.63 & 37.35 & 26.28 & 23.43 & 25.80 & 21.64 \\
\hline LOT $_{\text {opt }}$ & 39.95 & 27.25 & 24.19 & 26.21 & 22.65 & 38.38 & 26.42 & 23.43 & 25.86 & 21.63 \\
\hline LT $_{\text {opt }}$ & $\mathbf{4 0 . 2 6}$ & 26.94 & 23.88 & 26.01 & 22.59 & $\mathbf{3 8 . 8 7}$ & 26.20 & 23.16 & 25.72 & 21.49 \\
\hline \hline T6 & 37.21 & 27.10 & 24.15 & 26.16 & 22.66 & 32.59 & 25.88 & 23.31 & 25.38 & 21.65 \\
\hline T9 & 35.64 & 26.89 & 24.20 & 26.18 & 22.63 & 31.69 & 25.47 & 23.25 & 25.18 & 21.63 \\
\hline \hline P1 & 34.74 & $\mathbf{2 9 . 4 1}$ & $\mathbf{2 6 . 2 3}$ & $\mathbf{2 8 . 2 7}$ & $\mathbf{2 4 . 6 4}$ & 31.13 & $\mathbf{2 6 . 8 4}$ & $\mathbf{2 4 . 1 9}$ & $\mathbf{2 6 . 3 1}$ & $\mathbf{2 2 . 6 8}$ \\
\hline P2 & 38.38 & 29.33 & 26.02 & 28.05 & 24.28 & 35.76 & 26.37 & 23.30 & 25.90 & 22.14 \\
\hline P3 & 39.97 & 28.33 & 25.30 & 27.17 & 23.54 & 37.80 & 26.75 & 23.76 & 26.23 & 22.23 \\
\hline P4 & $\mathbf{4 0 . 2 6}$ & 27.02 & 24.03 & 26.20 & 22.86 & $\mathbf{3 8 . 8 7}$ & 26.02 & 22.98 & 25.69 & 21.74 \\
\hline
\end{tabular}

Table II tabulates reconstruction results in terms of PSNR if the images are transmitted without quantization. Clearly, a transform with a smaller MSE gives a higher PSNR. All LOTs produce about the same PSNR since they have the same MSE. Here, the $\mathrm{LT}_{\mathrm{opt}}$ does not outperform any of the LOTs. This is one of the reasons that orthogonal transforms are more popular in error-resilient coding so far. P1-P4 offer significantly higher PSNRs compared to the other transforms. By dynamically switching the post-filters, $\mathrm{P} 4$ consistently outperforms the
$\mathrm{LT}_{\mathrm{opt}}$. Several reconstructed portions of the Lena image are shown in Fig. 6. Although the images constructed with the DCT, the $\mathrm{LOT}_{\mathrm{opt}}$, and T9 have the same PSNR, the visual quality differs significantly since they have different reconstruction gains. Comparing to the DCT reconstruction, blocking artifacts are reduced in the $\mathrm{LOT}_{\text {opt }}$ construction, and even more suppressed in the T9 reconstruction. Although yielding a lower PSNR, the $\mathrm{LT}_{\mathrm{opt}}$ reconstruction is visually better than the DCT reconstruction. The P1-P4 reconstructions are superior than the others 
TABLE IV

RECONSTRUCTION RESULTS IN TERMS OF PSNR (CODED AT 0.25 bpp By L-CEB) UNDER VARIOUS LOSS PATtERNS

\begin{tabular}{c||c|c|c|c|c||c|c|c|c|c|c}
\hline \multicolumn{1}{c||}{ Transform } & S0 & S1 & S2 & S3 & S4 & S0 & S1 & S2 & S3 & S4 \\
\hline \hline \multicolumn{1}{l||}{} & \multicolumn{9}{|c||}{ Lena } \\
\hline DCT & 33.05 & 26.63 & 24.03 & 25.71 & 22.54 & 28.23 & 24.83 & 22.87 & 24.46 & 21.32 \\
\hline LOT $_{\text {opt }}$ & 33.61 & 26.72 & 24.02 & 25.82 & 22.57 & 29.78 & 25.29 & 23.02 & 24.90 & 21.43 \\
\hline LT $_{\text {opt }}$ & $\mathbf{3 4 . 3 4}$ & 26.55 & 23.78 & 25.73 & 22.56 & $\mathbf{3 0 . 0 3}$ & 25.18 & 22.83 & 24.84 & 21.34 \\
\hline \hline T6 & 28.29 & 26.23 & 23.69 & 25.57 & 22.64 & 25.43 & 23.65 & 22.35 & 23.39 & 21.10 \\
\hline T9 & 28.22 & 25.28 & 23.56 & 24.82 & 22.26 & 24.61 & 23.20 & 22.15 & 22.96 & 20.95 \\
\hline \hline P1 & 28.05 & 26.45 & 24.85 & 25.96 & 23.84 & 24.71 & 23.58 & 22.62 & 23.36 & 21.68 \\
\hline P2 & 31.97 & $\mathbf{2 8 . 0 6}$ & $\mathbf{2 5 . 5 2}$ & $\mathbf{2 7 . 1 3}$ & $\mathbf{2 4 . 0 5}$ & 27.06 & 24.47 & 22.62 & 24.19 & 21.69 \\
\hline P3 & 33.72 & 27.64 & 25.04 & 26.67 & 23.44 & 28.98 & $\mathbf{2 5 . 2 8}$ & $\mathbf{2 3 . 2 2}$ & $\mathbf{2 4 . 9 4}$ & $\mathbf{2 1 . 9 0}$ \\
\hline P4 & $\mathbf{3 4 . 3 4}$ & 26.63 & 23.92 & 25.89 & 22.81 & $\mathbf{3 0 . 0 3}$ & 25.00 & 22.65 & 24.80 & 21.47 \\
\hline
\end{tabular}
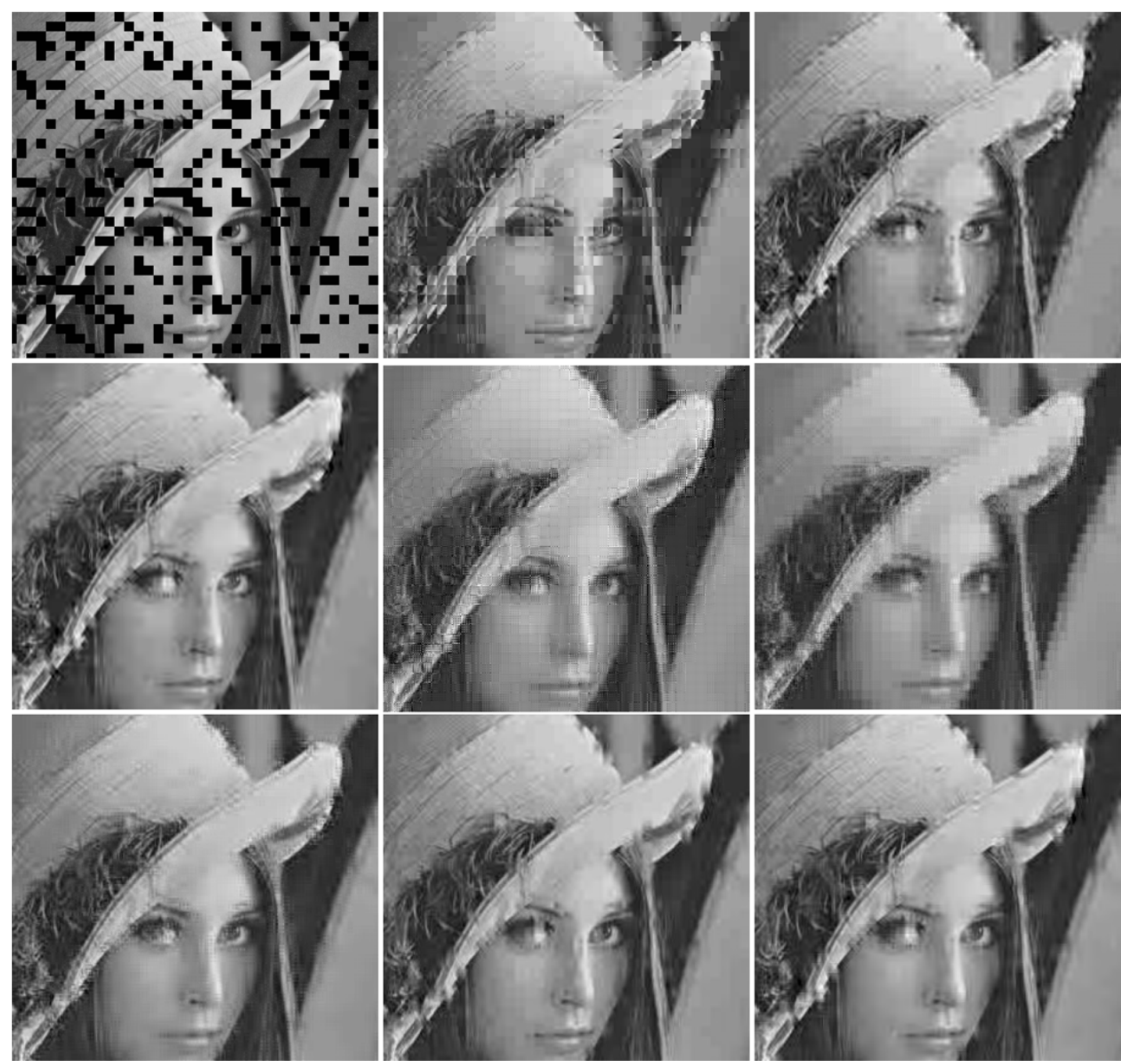

Fig. 7. Portions of reconstructed Lena image (coded at $0.25 \mathrm{bpp}$ ), suffering from $25 \%$ random loss. From left to right, top row: locations of loss; $\mathrm{DCT}$ ( $25.71 \mathrm{~dB}$ ); LOT $_{\text {opt }}(25.82 \mathrm{~dB})$. Middle row: $\mathrm{LT}_{\text {opt }}(25.73 \mathrm{~dB})$; T9 (24.82 dB); P1 (25.96 dB). Bottom row: P2 (27.13 dB); P3 (26.67 dB); P4 (25.89 dB).

in term of PSNRs and the distortion is almost imperceptible in the P1 reconstruction. Benefiting from dynamic post-filter switching, artifacts in the $\mathrm{P} 4$ reconstruction are visibly less annoying than those in the $\mathrm{LT}_{\mathrm{opt}}$ reconstruction.

However, the above simulation results only reflect reconstruction performance. In practice, we have to take coding performance into account. We use a JPEG-like block-based coder, L-CEB [4], to simulate an error-resilient block image coding system. The only differences between L-CEB and JPEG are:
1) LTs can take the place of the DCT in L-CEB and 2) L-CEB uses more advanced entropy coding. We assume that block loss occurs after decoding and blocks belonging to a specific loss pattern are reconstructed prior to performing the inverse transform. The coding and communication framework is fixed. Only different transforms are tested, ensuring the comparison is as fair as possible.

Tables III and IV list the reconstruction results in terms of PSNR for different transforms and different loss patterns when 

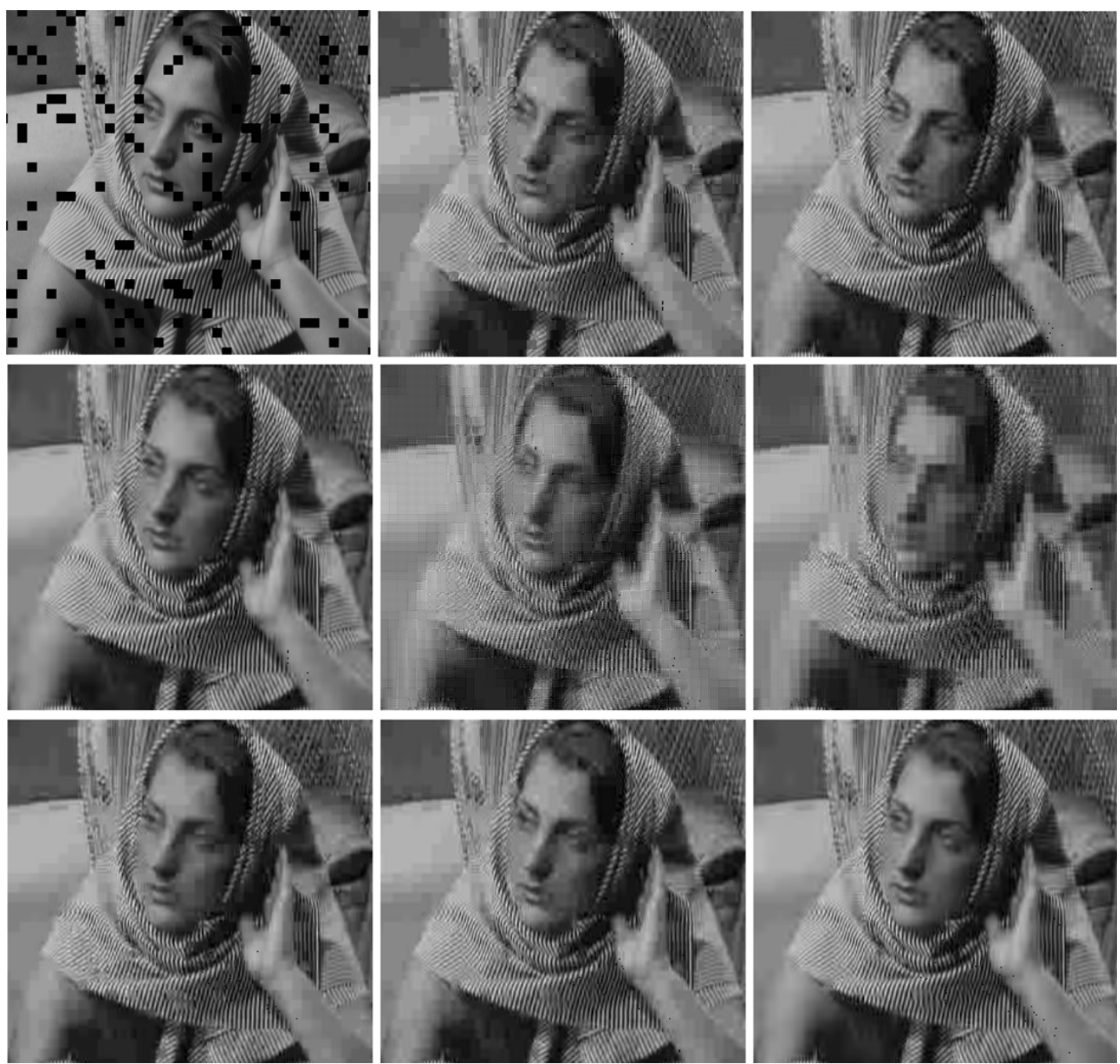

Fig. 8. Portions of reconstructed Barbara image (coded at $1 \mathrm{bpp}$ ), suffering from $10 \%$ random loss. From left to right, top row: locations of loss; DCT (29.69 dB); $\mathrm{LOT}_{\text {opt }}(29.61 \mathrm{~dB})$. Middle row: LT opt $_{2}(29.47 \mathrm{~dB})$; T9 (28.29 dB); P1 (28.62 dB). Bottom row: P2 (29.45 dB); P3 (30.05 dB); P4 (29.55 dB).

the images are coded by L-CEB at 1 and 0.25 bpp, respectively. The S0 columns only illustrate coding performance since there is no coefficient loss. Under this real-life communication environment, penalized by their bad coding performance, the overall performance of T6 and T9 in terms of PSNR is notably worse than that of the DCT, the $\mathrm{LOT}_{\mathrm{opt}}$ and the $\mathrm{LT}_{\mathrm{opt}}$ most of the time. On the other hand, our new designs P1-P4 generally offer much more reasonable performances. Again, $\mathrm{P} 4$ yields a consistent improvement over the $\mathrm{LT}_{\mathrm{opt}}$. Several reconstructed portions of Lena and Barbara are shown in Figs. 7 and 8. The reconstructs from P1-P3 are more pleasing than the others. The P4 reconstruction has less annoying artifacts than that of the $\mathrm{LT}_{\mathrm{opt}}$ and $\mathrm{LOT}_{\text {opt }}$ reconstruction.

Our experimental results well illustrate the superior overall performance of the proposed transforms P1-P4. Notice that P1 performs best at $1 \mathrm{bpp}$, but at $0.25 \mathrm{bpp}, \mathrm{P} 2$ or $\mathrm{P} 3$ offer better visual quality. This illustrates the fact that we should design error-resilient transforms according to the channel bandwidth as well as the expected loss pattern. With a higher loss percentage, reconstruction performance is more important. Another rule of thumb is that we should bias coding performance more at low bitrates. The reason is that at low bitrates most coefficients are quantized to zero and quantization errors become the dominant factor.

\section{CONCLUSION}

This paper presents the design of error-resilient pre/post-filters for the block DCT coding framework. The proposed solution can also be viewed as a fast and efficient method to design error-resilient LPs with low computational complexity. We demonstrate that each designed pre/post-filter pair provides a different tradeoff between compression and reconstruction performance. Hence, a pre/post-filter pair should be selected based on the channel characteristics as well as the desired bitrate. To conclude the paper, our original contributions are briefly summarized as follows.

- We revisit the block-based concealment problem from the new angle of adding pre- and post-filtering linear operators outside of the traditional DCT coding framework.

- Several novel error-resilient transforms are developed via: biorthogonal pre/post-filtering based on invertible linear operators and nonperfect-reconstruction pre/post-filtering based on dynamic switching at the 
decoder. Both approaches are proven to be capable of reducing the MSE of the reconstructed images comparing to the DCT and previous error-resilient LOT designs.

- Our solutions are highly practical. Every pre/post-filter design has a fast-computable separable algorithm. Standard compliance can be ensured since pre- and post-filtering operates outside of the existing coding/communication infrastructure. The proposed framework also leads to a much simpler transform optimization process. Most of the time, all free parameters are grouped together in one square matrix.

- Coding and communication simulations suggest that our current designs provide a significant performance gain over previous similar concealment approaches, both objectively and subjectively.

\section{ACKNOWLEDGMENT}

The authors would like to thank Dr. S. S. Hemami from Cornell University for providing the filter coefficients for LOTs T6-T9.

\section{REFERENCES}

[1] N. Ahmed, T. Natarajan, and K. R. Rao, "Discrete cosine transform," IEEE Trans. Comput., vol. C-23, no. 1, pp. 90-93, Jan. 1974.

[2] H. S. Malvar, Signal Processing With Lapped Transforms. Norwood, MA: Artech House, 1992.

[3] T. D. Tran, J. Liang, and C. Tu, "Lapped transform via time-domain pre- and post-processing," IEEE Trans. Signal Process., vol. 51, no. 6, pp. 1557-1571, Jun. 2003.

[4] C. Tu and T. D. Tran, "Context based entropy coding of block transform coefficients for image compression," IEEE Trans. Image Process., vol. 11 , no. 11 , pp. 1271-1283, Nov. 2002.

[5] T. D. Tran and C. Tu, "Lapped transform based video coding," in Proc. SPIE Applications of Digital Image Processing XXIV, San Diego, CA, Aug. 2001, pp. 319-333.

[6] Y. Wang and Q.-F. Zhu, "Error control and concealment for video communication: A review," Proc. IEEE, vol. 86, no. 5, pp. 974-997, May 1998.

[7] S. S. Hemami, "Reconstruction-optimized lapped orthogonal transforms for robust image transmission," IEEE Trans. Circuits Syst. Video Technol., vol. 6, no. 2, pp. 168-181, Apr. 1996.

[8] D. Chung and Y. Wang, "Multiple description image coding using signal decomposition and reconstruction based on lapped orthogonal transforms," IEEE Trans. Circuits Syst. Video Technol., vol. 9, no. 9, pp. 895-908, Sep. 1999.

[9] — , "Lapped orthogonal transform designed for error resilient image coding," IEEE Trans. Circuits Syst. Video Technol., no. 9, pp. 752-764, Sep. 2002.

[10] J. Liang and T. D. Tran, "DCT-based general structure for linear phase paraunitary filter banks," in Proc. IEEE Int. Conf. Acoustics, Speech, Signal Processing, vol. 2, Orlando, FL, May 2002, pp. 1169-1172.

[11] , "Further results on DCT-based general structure for linear phase paraunitary filter banks," in Proc. IEEE Int. Conf. Image Processing, vol. 2, Rochester, NY, Sep. 2002, pp. 681-684.
[12] W. Dai and T. D. Tran, "Regularity-constrained pre- and post-filtering for block-based coding and communication systems," IEEE Trans. Signal Process., vol. 51, no. 10, pp. 2568-2581, Oct. 2003.

[13] R. J. Clarke, "Application of image covariance models to transform coding," Int. J. Electron., vol. 56, no. 2, pp. 245-260, 1984.

[14] R. L. de Queiroz and K. R. Rao, "The extended lapped transform for image coding," IEEE Trans. Image Process., vol. 4, no. 6, pp. 828-832. Jun. 1995.

[15] P. Haskell and D. Messerschmitt, "Reconstructing lost video data in a lapped orthogonal transform based code," in Proc. Int. Conf. Acoust. Speech, Signal Processing, vol. C-23, Albuquerque, NM, 1990, pp. 1985-1988.

Chengjie Tu (S'02-M'04) received the B.E. and M.E. degrees from University of Science and Technology of China in 1994 and 1997, respectively, and the M.S.E. and Ph.D. degrees from The Johns Hopkins University, Baltimore, MD, in 2002 and 2003, respectively.

He is currently with the Video Codec Group, Microsoft Digital Media Division, Redmond, WA. His current research interests include image/video compression, multirate signal processing, and error control and concealment for image/video communication.

Trac D. Tran (S'94-M'98) received the B.S. and M.S. degrees from the Massachusetts Institute of Technology, Cambridge, in 1993 and 1994, respectively, and the Ph.D. degree from the University of Wisconsin, Madison, in 1998, all in electrical engineering.

In July 1998, he joined the Department of Electrical and Computer Engineering, The Johns Hopkins University, Baltimore, MD, where he is currently an Associate Professor. His research interests are in the field of digital signal processing, particularly in multirate systems, filter banks, transforms, wavelets, and their applications in signal analysis, compression, processing, and communications. He was the Co-Director (with Prof. J. L. Prince) of the 33rd Annual Conference on Information Sciences and Systems, Baltimore, MD, in March 1999. In the summer of 2002, he was an ASEE/ONR Summer Faculty Research Fellow at the Naval Air Warfare Center Weapons Division (NAWCWD), China Lake, CA.

Dr. Tran received the National Science Foundation CAREER award in 2001. He currently serves as an Associate Editor for the IEEE TRANSACTIONS ON Signal Processing and the IEEE TRAnsactions on IMAge Processing. He is also a member of the IEEE Technical Committee on Signal Processing Theory and Methods (SPTM TC).

Jie Liang (S'99-M'04) received the B.E. and M.E. degrees from Xi' an Jiaotong University, China, in 1992 and 1995, respectively, the M.E. degree from the National University of Singapore in 1998, and the Ph.D. degree from The Johns Hopkins University, Baltimore, MD, 2003.

He is now an Assistant Professor at the School of Engineering Science, Simon Fraser University, Burnaby, BC, Canada. From June 2003 to May 2004, he was with the Video Codec Group, Microsoft Digital Media Division, Redmond, WA. His research interests include multirate signal processing, image/video compressions, and digital communications. 